\title{
Aspectos del cultivo y la fisiología de la feijoa \\ (Acca sellowiana [Berg] Burret). Una revisión
}

ISSN 0122-8420

elSSN2539-0899

Fecha de recepción:07 de Julio de 2020

Fechadeaceptación: 11 de aqosto de 2020
Gerhard Fischer - 1

Alfonso Parra-Coronado $\cdot 2$

Helber Enrique Balaguera-López • 3

DOI: https://doi.org/10.19053/01228420.v17.n3.2020.11386

\section{Resumen}

La feijoa es un frutal con gran potencial para el consumo humano, que se cultiva entre los 1800 y $2700 \mathrm{msnm}$ en Colombia. Está bien adaptada a temperaturas bajas, resistiendo por corto tiempo temperaturas $<0{ }^{\circ} \mathrm{C}$, mientras que temperaturas $>32{ }^{\circ} \mathrm{C}$ afectan su floración. La temperatura base para botón floral a antesis es $2,89{ }^{\circ} \mathrm{C}$, de antesis a cuajamiento del fruto es de $3,04{ }^{\circ} \mathrm{C}$ y del cuajamiento del fruto a cosecha, $1,76{ }^{\circ} \mathrm{C}$. El árbol crece muy bien en suelos aluviales, profundos y ricos en materia orgánica. En la poda del árbol es importante la formación en cono con tres niveles de ramas agobiadas y la eliminación de los chupones, para fomentar así la floración y la longevidad del árbol. En los pocos estudios fisiológicos se encontró una tasa fotosintética baja (en promedio entre 4 y $6 \mu \mathrm{mol} \mathrm{CO}_{2} \mathrm{~m}^{-2} \mathrm{~s}^{-1}$ ), con gran demanda energética durante la floración, mientras la transpiración aumenta con la irradiancia, la temperatura y la disminución de la humedad relativa, mostrando un mayor número de estomas foliares en el estrato superior de la copa $\left(91 / \mathrm{mm}^{2}\right)$, comparado con el inferior $\left(78 / \mathrm{mm}^{2}\right)$. El inicio de la etapa reproductiva está favorecida por las condiciones más secas y por la fertilización con $\mathrm{KH}_{2} \mathrm{PO}_{4}$ al $0.5 \%$. En Colombia, frutos de mayor calibre y producciones más altas se logran a través de la polinización por la mirla negra (Turdus fuscater). El fruto necesita entre 120 y 150 días para desarrollarse y se presentan producciones entre 30 y $40 \mathrm{~kg} / a ́ r b o l ~\left(\geq 20 \mathrm{t} \mathrm{ha}^{-1}\right)$. El color verde del fruto en todos sus estados dificulta la determinación del grado de madurez.

Palabras clave: ecofisiología, nutrición, poda, transpiración, inducción floral, polinización, desarrollo fruto.

\section{Aspects of Crop and Physiology of Feijoa}

\section{(Acca sellowiana [Berg] Burret). A Review}

\begin{abstract}
Feijoa is a fruit tree with great potential for human consumption, cultivated between 1,800 and 2,700 m.a.s.l. in Colombia. It is well adapted to cold temperatures, resisting temperatures $<0{ }^{\circ} \mathrm{C}$ for a short time, while temperatures $>32^{\circ} \mathrm{C}$ affect its flowering. It has base temperatures for its state from flower bud to anthesis with $2.89{ }^{\circ} \mathrm{C}$, from anthesis to fruit set with $3.04{ }^{\circ} \mathrm{C}$ and from fruit set to harvest $1.76{ }^{\circ} \mathrm{C}$. The tree thrives well in in alluvial soils, deep and rich in organic matter ones. In the tree pruning, the cone formation with three levels of horizontal bent branches and the elimination of suckers are important, thus promoting flowering and longevity of the tree. In the few physiological studies, a low photosynthetic rate (on average between 4 and $6 \mu \mathrm{mol} \mathrm{CO} \mathrm{m}^{-2} \mathrm{~s}^{-1}$ ) was found, with high energy demand during flowering, while the transpiration rate of the crown increases with irradiance, temperature and the decrease in relative humidity, showing a greater number of leaf stomata in the upper layer of the crown $\left(91 / \mathrm{mm}^{2}\right)$, compared to the lower layer $\left(78 / \mathrm{mm}^{2}\right)$. The beginning of the reproductive stage is favored by dry conditions, as well as potassium fertilization with $0.5 \% \mathrm{KH}_{2} \mathrm{PO}_{4}$. In Colombia, fruits of greater caliber and higher productions are achieved through cross-pollination by blackbirds (Turdus fuscater). The fruit needs about 120 to 150 days to develop and there are productions between 30 and $40 \mathrm{~kg} / \mathrm{tree}$ ( $\geq 20 \mathrm{t}$ $\left.\mathrm{ha}^{-1}\right)$. The green color of the fruit in all its stages makes it difficult to determine its maturity state.
\end{abstract}

Keywords: ecophysiology, nutrition, pruning, transpiration, floral induction, pollination, fruit development.

IPhD. Facultad de Ciencias Agrarias, Universidad Nacional de Colombia. Bogotá, Colombia. gfischer@unal.edu.co https://orcid.org/0000-0001-8101-0507 2Ph.D. Facultad de Ingeniería, Universidad Nacional de Colombia. Bogotá, Colombia. aparrac@unal.edu.co e https://orcid.org/0000-0001-9045-2083

3Ph.D. Facultad de Ciencias Agrarias, Universidad Nacional de Colombia. Bogotá, Colombia. hebalagueral@unal.edu.co

chttps://orcid.org/0000-0003-3133-0355 


\section{Introducción}

En los últimos años se ha evidenciado un incremento importante en la investigación en frutales andinos (Naizaque et al., 2014; Gallo et al., 2018; Fischer \& Melgarejo, 2020). La feijoa (Acca sellowiana [O. Berg] Burret) es oriunda de América del Sur, de la región del sur de Brasil, Uruguay, zona alta de Paraguay occidental y el noreste de Argentina (Parra-Coronado \& Fischer, 2013). Es mayormente sembrada en Colombia, Nueva Zelanda, Georgia y Ucrania, y en áreas menores en Estados Unidos, Australia, Nueva Zelanda, Turquía y China, con plantaciones crecientes en Brasil (Sachet et al., 2019), por lo cual Donazzolo et al. (2019) destacan su plasticidad y adaptación a condiciones ambientales muy diversas, hecho demostrado por esta distribución en zonas y climas tan variados.

También en Colombia la feijoa muestra una adaptación a una amplia gama de condiciones agroecológicas (Naizaque et al., 2014). Encuentra las condiciones óptimas para su producción comercial entre 1800 y 2700 msnm (Quintero, 2012) y los departamentos de mayor producción en 2018 fueron Boyacá (242 ha; 2372 t) y Cundinamarca (55 ha; 368 t) para un total de 307 ha y $2765 \mathrm{t}$ en el país (Agronet, 2020). El cultivo en los trópicos puede presentar cosechas continuas a lo largo del año, mientras que, según Quintero (2012), en las regiones subtropicales y estacionales se obtiene solamente una cosecha anualmente.

El comportamiento fisiológico de un cultivo y la calidad de sus frutos dependen de las características de cada especie, las condiciones ambientales y el manejo del cultivo (Fischer et al., 2018). Para el caso de la feijoa, teniendo grandes expectativas comerciales y socioeconómicas, además por su valor nutricional y medicinal (Peña \& Cabezas, 2014), el objetivo de esta revisión es informar sobre el estado actual de los conocimientos en aspectos del cultivo y su fisiología, como son la ecofisiología, nutrición, poda, inducción floral, polinización y desarrollo del fruto, entre otros, que ayudan a optimizar el manejo agronómico de esta especie.

\section{Botánica}

Esta planta pertenece a la familia Myrtaceae, igualmente como la guayaba (Perea et al., 2010), teniendo en cuenta que esta familia, con sus 121 géneros, es clasificada dentro de las familias comerciales de frutas más importantes del mundo (Farias et al., 2020). La feijoa crece como un arbusto o árbol pequeño hasta una altura máxima de 3,5 a $5 \mathrm{~m}$ y se destaca como un frutal promisorio perenne y longevo (Fischer, 2003) que se conoce también en otros países como planta ornamental (Martínez-Vega et al., 2008).

El sistema radicular de ésta planta es fibroso y superficial con muchas raicillas absorbentes (Perea et al., 2010), que puede, en suelos aluviales, extenderse entre 1,5 y $2,0 \mathrm{~m}$ horizontal y 0,4 a $0,5 \mathrm{~m}$ verticalmente (Fischer, 2003). La corteza rojiza-cobriza del tallo y de las ramas gruesas se desprende fácil superficialmente en forma de placas. Las hojas de forma elíptica-ovalada son pequeñas, de $6 \times 4 \mathrm{~cm}$, con el haz de color verde brillante y el envés blanquecino (Fischer, 2003).

En las axilas de las hojas en ramas de año se desarrollan las flores hermafroditas, con 4-6 sépalos verde-grisáceos y una corola de 4 pétalos blancos, presentando en el centro 70 - 80 estambres de filamentos rojos oscuros con sus 
anteras blancas (Duarte \& Paull, 2015; Fischer, 2003). Algunos cultivares son autofértiles, pero existe también la polinización cruzada a través de insectos o pájaros (Quintero, 2012). Curiosamente, se pueden comer los pétalos de las flores que presentan altas concentraciones de antocianinas, flavonoides, fenoles, antioxidantes y sólidos solubles (Amarante et al., 2019). El fruto es clasificado como una baya (contiene 20-40 semillas), es de formas redondas hasta ovoides, de color verde brillante a verde grisáceo, con piel lisa a rugosa, $3-5 \mathrm{~cm}$ de diámetro y 4-10 $\mathrm{cm}$ de longitud, y pesa entre 20 y $250 \mathrm{~g}$, según variedad (Amarante \& Santos, 2011).

Ramírez y Kallarackal (2018) describieron en dos cultivares de feijoa, seis estados fenológicos diferentes siguiendo la escala de la BBCH (Biologische Bundesanstalt, Bundessortenamt und CHemische Industrie) como: (1) desarrollo del botón, (2) desarrollo foliar, (3) desarrollo del tallo, (4) emergencia de flores, (5) floración y (6) desarrollo del fruto.

Existe un interés creciente de utilizar la feijoa para consumo humano debido a su calidad comestible muy atractiva y sus efectos sobre la salud. El fruto es una fuente importante de fibra, azúcares, polifenoles, vitamina $\mathrm{C}$ y minerales, especialmente potasio (Zhu, 2018). Se consume principalmente en fresco (con un sabor dulce y muy aromático), además es apto principalmente para jugos, jaleas, chutneys, bebidas, yogurts y helados (Mitra, 2010). Tiene importancia medicinal por su actividad antibacteriana, antioxidante y antialérgica (Amarante \& Santos, 2011); también las hojas se pueden usar para infusiones con el fin de curar disentería y cólera (Thorp, 2008).

\section{Ecofisiología}

Debido a sus zonas de origen, el árbol de la feijoa es capaz de resistir temperaturas tan bajas como $-4{ }^{\circ} \mathrm{C}$; $\sin$ embargo, las flores y frutos pequeños pueden quemarse con temperaturas cercanas a $-1^{\circ} \mathrm{C}$ cuando se exponen por más de una hora, y también se afectan sus frutos maduros por pardeamiento interno (Pachón \& Quintero, 1992; Schotsmans et al., 2011).

Autores como CRFG (1996) y Sharpe et al. (1993) recomiendan para maximizar la floración la acumulación de 50 y 100-200 h a unos $7{ }^{\circ} \mathrm{C}$, respectivamente, que mejorarian la producción de flores para esta especie; sin embargo, este efecto no se ha encontrado en las zonas tropicales, lo que exige hacer un estudio más profundo sobre este requerimiento de frío. Quintero (2003) constata que en los trópicos la feijoa más bien reacciona para una mejor inducción floral y floración al cambio de las temporadas secas y húmedas y también a la poda.

En el caso de las zonas tropicales, Barrero (1993) recomienda para cultivos comerciales de la feijoa temperaturas dentro de un rango de 13 a $21^{\circ} \mathrm{C}$ (promedio $16{ }^{\circ} \mathrm{C}$ ), como las más favorables para esta especie. Sin embargo, temperaturas altas $\left(>32^{\circ} \mathrm{C}\right)$ y condiciones muy secas durante la floración pueden afectar la polinización y el cuajado de frutos (Duarte \& Paull, 2015).

Parra-Coronado et al. (2015a) encontraron las temperaturas base (las mínimas en las cuales inicia el crecimiento) para diferentes etapas de la fenología reproductiva del cultivar 'Quimba' para la etapa botón floral a antesis con 2,89 ${ }^{\circ} \mathrm{C}$, de antesis a cuajamiento del fruto $3,04{ }^{\circ} \mathrm{C}$, del cuaje fruto a cosecha $1,76{ }^{\circ} \mathrm{C}$, lo que destaca la adaptación de este cultivar a las condiciones frías del trópico alto colombiano.

Las etapas de floración, polinización, cuajamiento y llenado del fruto tienen sus mejores resultados a libre exposición a la radiación solar, con unas $1500 \mathrm{~h}$ de brillo solar/año o más (Duarte \& Paull, 2015), teniendo en cuenta que la 
planta se adapta a plena luminosidad cuando el ambiente no es muy seco o caliente (Fischer, 2003). En zonas con menos de $1500 \mathrm{~h}$ de brillo solar/año, Duarte y Paull (2015) sugieren que las demás condiciones ambientales deben compensar esta deficiencia luminosa y además, la geometría de la copa debe ser adaptada para una intercepción eficiente de luz a través de la formación de la copa y las prácticas de la poda (ver capítulo poda). También en lulo se encontró que el efecto de la sombra no es tan limitante para el crecimiento como otros factores estresantes, por ejemplo el anegamiento (Sánchez-Reinoso et al., 2019).

Condiciones de sombra causan el alargamiento de los entrenudos de las ramas, además Martínez-Vega et al. (2008) encontraron en feijoas formadas con un líder central, que los frutos en las zonas sombreadas del árbol (en la base y la parte interna de la copa) tuvieron un color verde más intenso, mientras los frutos en la periferia superior de la copa desarrollaron una mayor madurez, pero con un peso menor que los ubicados en la parte media de la copa (Fischer \& Parra-Coronado, 2020).

La altitud más adecuada para las plantaciones comerciales en Colombia se encuentra entre 1800 y 2700 msnm (Parra-Coronado et al., 2019), teniendo las mejores zonas productivas en regiones entre 2100 y 2600 msnm (Duarte \& Paull, 2015). Por debajo de 1800 msnm existe un peligro inminente por la mosca de la fruta (Anastrepha sp.), sin embargo, también puede atacar hasta elevaciones de 2600 msnm, como reportó Quintero (2012) en Tibasosa (Boyacá).

La altitud, por sus condiciones específicas como temperatura, radiación, humedad relativa, presión parcial de gases y viento (Fischer \& Orduz-Rodríguez, 2012), influye en la calidad de las feijoas. En Cundinamarca, Parra-Coronado et al. (2019) compararon la calidad de las feijoas 'Quimba' en cosecha y poscosecha, provenientes de una altitud de 1800 msnm (localidad: San Francisco de Sales; temperatura media del estudio $18,3^{\circ} \mathrm{C}$ ) y una de $2580 \mathrm{msnm}$ (localidad: Tenjo; temperatura media de $12,3^{\circ} \mathrm{C}$ ) y encontraron que los frutos en la menor altitud crecieron más rápido (155 días después de plena floración) y presentaron menos sólidos solubles totales $\left(11,4^{\circ}\right.$ Brix $)$ y un peso fresco menor (entre 30 y $46 \mathrm{~g}$ ) en el momento de la cosecha; mientras que el crecimiento de los frutos a mayor altitud tomó más tiempo (180 días después de plena floración) y estos presentaron mayor peso (entre 38 y 99 g) y más grados Brix $\left(12,6^{\circ}\right.$ Brix $)$, debido probablemente a la mayor radiación solar registrada, mientras la acidez total titulable no fue influenciada por la elevación (Parra-Coronado et al., 2015b).

La influencia de estas dos altitudes en la calidad poscosecha de los frutos de feijoa almacenando a $5{ }^{\circ} \mathrm{C}$ (humedad relativa media de $76 \%$ ) y a temperatura ambiente de $18{ }^{\circ} \mathrm{C}$ (humedad relativa media de $87 \%$ ) se manifestó de manera similar a lo presentado en el momento de la cosecha, hecho que indica que la calidad poscosecha depende directamente de la calidad que tengan los frutos en el momento de la recolección, lo cual se reflejó en que los frutos producidos a menor altitud presentaron menor valor de grados Brix, menor pérdida de firmeza y mayor valor de acidez titulable y pérdida de peso. Además, los frutos almacenados a menor temperatura son menos dulces y muestran menor pérdida de peso y de firmeza (Parra-Coronado et al., 2018).

Las plantaciones comerciales de feijoa exigen entre 700 y $1200 \mathrm{~mm}$ de precipitación anual, tolerando hasta unos 2000 $\mathrm{mm}$ año-1 en el caso de una suficiente luminosidad y humedad relativa del $70 \%$, lo que garantiza una producción continua de frutos de calidad (Fischer \& Parra-Coronado, 2020). El tamaño y la calidad del fruto dependen de los suministros adecuados y regulares del agua. Sin embargo, el exceso de agua y el incorrecto manejo del drenaje pueden ser causantes de pérdidas en la cosecha (Fischer et al., 2012). En caso contrario, las sequías prolongadas pueden inducir caídas foliares, de flores y de frutos o una maduración prolongada de estos (Jackson \& Looney, 1999). Con el fin de mejorar las producciones, CRFG (1996) recomienda aplicar el agua constantemente, con preferencias durante la floración y fructificación, así como aporcar las bases del tallo para cubrir las raíces superficiales. Debido a la poca información sobre el riego y los requerimientos hídricos de la feijoa, Duarte y Paull (2015) recomiendan aplicar 
el riego (preferiblemente por goteo o microaspersión) solamente en épocas muy secas o en zonas de poca lluvia, para garantizar una buena producción y calidad de los frutos.

Peña y Cabezas (2014) realizaron estudios de riego deficitario, para lo cual regaron plantas de 3 años de feijoa (cv. Tibasosa a $15{ }^{\circ} \mathrm{C}$ y $850 \mathrm{~mm}$ de precipitación) a $100 \%, 75 \%, 50 \%, 25 \%$ y $0 \%$ (testigo) durante 406 días, encontrando que el riego no afectó la altura de la planta, pero el déficit hídrico ( $0 \%$ riego adicional) disminuyó significativamente el número de hojas, área foliar e índice de área foliar, pero no el número de ramas. Este estudio también mostró que el riego a $25 \%$ produjo el mayor número de botones florales a los 263 y 320 días de la evaluación, lo que indica la importancia de un crecimiento vegetativo reducido por una época más seca, ya que favorece la inducción floral en varios frutales en condiciones tropicales (Fischer et al., 2012). Quintero (2014) afirma que una época seca beneficia más la floración en feijoa que una más húmeda.

Siendo la feijoa resistente al viento (Duarte \& Paull, 2015), los vientos suaves aseguran el secado de hojas, flores y frutos mojados y activan la apertura de los estomas después de una lluvia, mientras en días de alto calor refrescan la copa del árbol y aseguran la renovación del $\mathrm{CO}_{2}$, usado en la fotosíntesis; por el contrario, vientos fuertes pueden causar la rotura de ramas, caída de flores, hojas y frutos (Fischer \& Orduz-Rodríguez, 2012; Arias-Cruz et al., 2016).

\section{Nutrición}

La feijoa no es muy exigente con la calidad del suelo, prospera en los de tipo arenoso hasta arcilloso, con los mejores resultados en suelos aluviales, profundos y ricos en nutrientes y materia orgánica, mientras los franco-arenosos a franco-arcillosos fomentan el desarrollo radicular de la planta (Quintero, 2014). Para los mejores rendimientos de la feijoa, Thorp (2008) recomienda suelos bien drenados y ligeramente ácidos con un $\mathrm{pH}$ entre 6,0 y 6,5. En suelos con un $\mathrm{pH}<6,0$ Quintero (2014) sugiere adicionar $2 \mathrm{~kg}$ de cal dolomita al hoyo de la siembra.

Duarte y Paull (2015) afirman que la feijoa es un árbol de crecimiento lento con un requerimiento de nitrógeno relativamente bajo en relación con el de potasio y fósforo, por lo cual Thorp (2008) sugiere aplicar fertilizantes de lenta liberación de nutrientes. Morley-Bunker (1999) recomienda aplicar 25-30 kg ha-1 de nitrógeno el primer año, aumentando gradualmente la cantidad anual hasta unos $120 \mathrm{~kg}$ en el décimo año; en cuanto al fósforo, aconseja abonar con $40 \mathrm{~kg} \mathrm{ha}^{-1}$ en el primer año incrementando hasta $80 \mathrm{~kg}$, finalmente para el caso del potasio aplicar $20 \mathrm{~kg}$ $\mathrm{ha}^{-1}$ durante el primer año llegando hasta $100 \mathrm{~kg}$ en plantas de producción. Sin embargo, cualquier aplicación según "receta" no es recomendable, si no se exige antes de la plantación y cada segundo año, mínimo un análisis del suelo y un análisis foliar (Quintero, 2012).

Problemas en la calidad de frutos se han presentado en suelos con una relación $\mathrm{Ca} / \mathrm{Mg} \geq 10$, desarrollando en estas condiciones frutos con pigmentaciones pardas, agrietados, cloróticos y con poca vida en poscosecha (<3 días), por lo cual Quintero (2003) recomienda aplicar $3 \mathrm{~kg} /$ planta/año de cal dolomita. Igualmente, en suelos con $\mathrm{pH}<5.0 \mathrm{y}$ deficientes en nitrógeno pueden desarrollarse frutos cloróticos, no aptos para el almacenamiento, por lo cual Quintero (2014) también sugiere enmiendas con cal dolomita ( $3 \mathrm{~kg} /$ planta/año) y suministrar al suelo fertilizantes ricos en nitrógeno (17-6-18-6, 18-18-18 o urea), al menos $500 \mathrm{~g}$ cada cuatro meses.

Buenos resultados han mostrado las aplicaciones de abonos orgánicos como complemento de la fertilización química en Colombia, por ejemplo, incorporar $10 \mathrm{~kg}$ de compost y gallinaza por planta y año, adicionando microorganismos 
eficientes para su mejor descomposición; además la materia orgánica ha mostrado grandes ventajas en terrenos muy inclinados, frenando la erosión (Quintero, 2014).

Buitrago (2017) cultivó plántulas de feijoa de nueve meses de edad con deficiencia de N, P, K, Ca, Mg y B, y encontró que los primeros síntomas se presentaron a los 39 días de iniciados los tratamientos para el caso del calcio, mientras que para el nitrógeno, fósforo, potasio y magnesio se expresaron síntomas apenas después de 60 días y para el boro a partir de 88 días. Las deficiencias de $\mathrm{N}$ y $\mathrm{Ca}$ fueron las que afectaron en mayor medida a las distintas variables de crecimiento y del contenido de clorofilas.

La feijoa muestra cierta tolerancia a la salinidad, como lo comprobaron Casierra-Posada y Rodríguez (2006), quienes adicionaron al sustrato de las materas ubicadas bajo invernadero 0,20, 40,60 y $80 \mathrm{mM}$ de $\mathrm{NaCl}$ en forma creciente, que no causó problemas en la distribución de biomasa y la producción de las plantas, las cuales reaccionaron con una disminución de la transpiración. Sin embargo, Duarte y Paull (2015) constataron que la planta es moderadamente tolerante a la salinidad, la cual reduciría el crecimiento y la producción de frutos. Por esta situación, son necesarios más estudios sobre el efecto de la salinidad en feijoa.

\section{Poda}

La poda de los árboles de feijoa es un manejo esencial en las plantaciones tecnificadas (Quintero, 2014). No es solamente crucial para la inducción floral, sino también mejora la productividad y calidad de los frutos, además es fundamental para el control fitosanitario (Quintero, 2003).

\section{A. Podas de formación}

Aparte del crecimiento libre en forma arbustiva, en las plantaciones tecnificadas Quintero (2003) propone podar los árboles en forma de cono, que consiste en un eje central del cual se desprenden 12 ramas primarias -tres pisos con cuatro ramas cada uno- y agobiadas formando ángulos de $90^{\circ}$ con respecto al líder principal, iniciando esta labor a partir de los 18 meses después de la plantación, dejando la primera ramificación en $50 \mathrm{~cm}$. La ventaja de este sistema u "ortopedia", usando estacas y pitas, es la mayor iluminación que permite la buena distribución de los frutos sobre todas las ramas primarias y secundarias, además que la altura de los árboles se mantiene hasta 2,5 $\mathrm{m}$ formando así frutos de alto calibre y calidad, sin disminuir la cosecha (Quintero, 2014).

\section{B. Podas de producción y mantenimiento}

Este tipo de podas permite programar las cosechas (Quintero, 1993) y consisten en eliminar las ramas secundarias y terciarias que crecen verticalmente (chupones), mientras con el agobio de las ramas en posición horizontal se controla el crecimiento para que la planta no invada las calles o se enrede con la planta siguiente. Además, esta poda formando ramas agobiadas, fomenta la inducción floral por una mejor distribución de las hormonas, especialmente las citoquininas que se mueven hacia la parte superior disminuyéndose la producción de auxinas que migran hacia la parte inferior de la rama (Casierra-Posada \& Fischer, 2012). En esta poda no se remueven las ramas muy finas (que mayormente cuelgan en el interior de la copa), porque estas son importantes para la próxima cosecha (Duarte \& Paull, 2015). 
La regulación de cosechas no es solamente influenciada por las pocas precipitaciones durante la floración, sino también por las podas que pueden inducir rebrotes que forman nuevas flores, pero solamente en árboles de una buena fertilidad que muestran botones florales, flores y frutos recientemente cuajados (Quintero, 2003). En estos casos, los rebrotes que se estimulan con estas podas se forman acompañados de una buena cantidad de nuevos botones florales, pero esta respuesta favorable no se presentará en árboles de baja fertilidad reproductiva (Quintero, 2014).

Sánchez-Mora et al. (2017) hicieron una poda a través del raleo de ramas poco productivas y las más vigorosas en el centro de la copa de árboles arbustivos de feijoa en Sao Joaquim (SC, Brasil), lo que causó una disminución del índice de área foliar y el número de frutos, pero fomentó el tamaño de los frutos cosechados.

\section{Vl. Fotosíntesis y Transpiración}

Las mediciones de la fotosíntesis en feijoa son muy escasas. En un estudio en la costa oriental de Sicilia, la tasa fotosintética de la variedad Mammouth durante los meses de julio a septiembre osciló, en promedio, entre 4 y 6 umol $\mathrm{CO}_{2} \mathrm{~m}^{-2} \mathrm{~s}^{-1}$, siempre menor que la fotosíntesis del aguacate (Germanà \& Continella, 2004).

Estudios de la transpiración foliar en una plantación comercial de feijoa en Tibaná (Boyacá, Colombia) en los dos estratos de la copa mostraron que el estrato superior se caracterizó por una tasa de transpiración y número de estomas foliares más altos ( $\left(91 / \mathrm{mm}^{2}\right.$ vs. $78 / \mathrm{mm}^{2}$ del inferior) (Naizaque et al., 2014). En general, estos autores encontraron que la transpiración se incrementó a medida que aumentó la temperatura (Figura 1) y la irradiancia, además las hojas perdieron más agua cuando se disminuyó la humedad relativa en la copa.

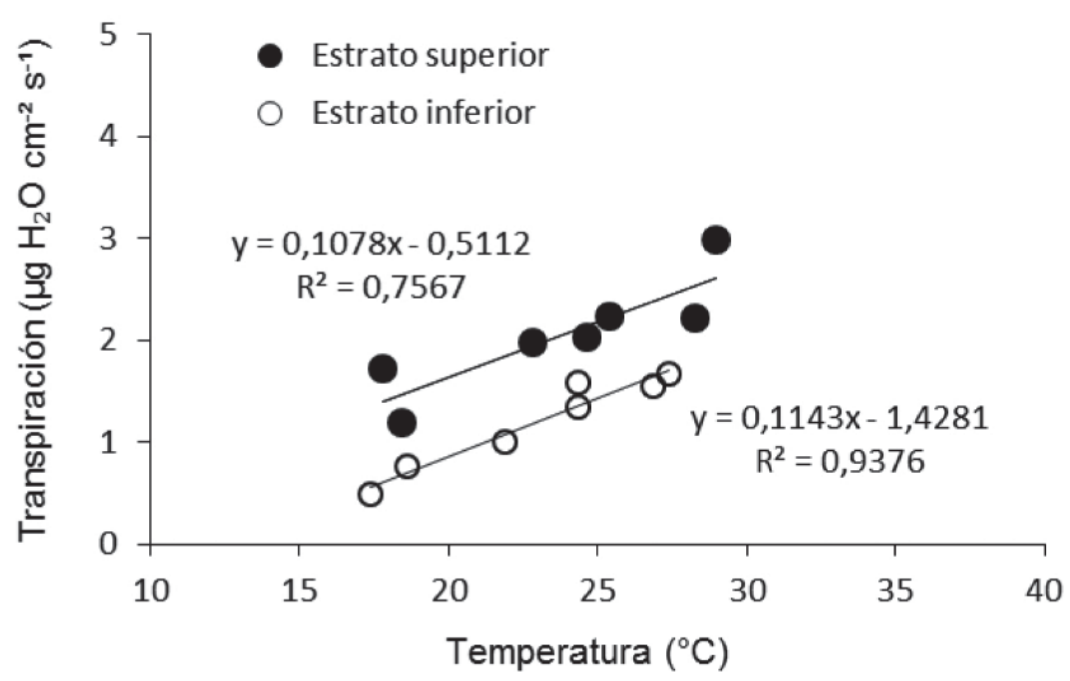

Figura 1. Efecto del aumento de la temperatura sobre la transpiración foliar en los estratos superior e inferior de la copa de árboles de feijoa.

Fuente: Naizaque et al. (2014). Con permiso de la Revista UDCA Actualidad \& Divulgación Científica.

En una comparación de varios procesos ecofisiológicos entre la feijoa, la chirimoya y el aguacate, Germanà y Continella (2004) encontraron que la feijoa desarrolla una baja eficiencia en el uso del agua (WUE, por sus siglas en 
inglés), acompañada por elevadas tasas de respiración, particularmente en la fase de la floración que exige una tasa de energía muy alta. Igualmente, la transpiración foliar de la feijoa fue más alta por su reducida resistencia estomática que generó solamente una WUE de 1/3 en comparación con la chirimoya y el aguacate (Germanà \& Continella, 2004).

\section{Inducción Floral}

Las plantas de feijoa ya muestran después de 2 años de la plantación las primeras flores y entran después de los 4-6 años en una floración plena (Fischer, 2003). Como ya se mencionó, las condiciones secas fomentan la inducción de flores, la floración y el cuajamiento de frutos (Fischer \& Parra-Coronado, 2020). Así mismo el amarre de las ramas laterales hasta una posición horizontal genera una distribución de las hormonas más favorable, promoviendo la inducción floral (Casierra-Posada \& Fischer, 2012).

García et al. (2008a) comprobaron que aplicaciones con $\mathrm{KH}_{2} \mathrm{PO}_{4}$ al $0.5 \%$ en el clon 41 mejoran la fertilidad, en este caso, con botones florales, flores y frutos cuajados (Quintero, 2003), incrementan la floración en el cultivar 41, aumentando el número de los botones florales inducidos y las flores formadas; además este producto indujo el mayor número de frutos cuajados (García et al., 2008b) y cosechados (Combariza et al., 2008). Este resultado hizo que muchas fincas ahora aumentaran sus aplicaciones en fertilizantes foliares ricos en fósforo para mejorar la floración (Quintero, 2014).

\section{Polinización}

Para garantizar el máximo de las flores polinizadas, Quintero (2014) encontró que el mejor método para optimizar la polinización es el establecimiento en un $67 \%$ de un mismo cultivar, en tanto el 33\% restante se siembra con otras variedades; por lo cual, este autor recomienda que después de cada dos hileras continuas del cultivar principal se establezca una hilera completa con la variedad polinizadora. La razón es la alta autoincompatibilidad de muchas variedades de feijoa (Thorp, 2008). Por ejemplo 'Mammoth' es considerado autofértil debido a su porcentaje de cuajamiento de frutos (55 \%), al contrario de 'Triumph', que muestra una barrera para su autopolinización (Ramírez \& Kallarackal, 2017).

Quintero (2012) reportó que la polinización cruzada puede aumentar el porcentaje de frutos cuajados, entre un 80 y 90 $\%$, comparado con la autofecundación que produce apenas la mitad de los cuajados. Además, este autor afirma que los frutos procedentes de una polinización cruzada muestran un calibre mayor que los producidos por autofecundación, lo que confirma lo indicado por California Rare Fruit Growers (CRFG, 1996) respecto a que las cosechas pequeñas son normalmente el resultado de una polinización no adecuada.

Los agentes polinizadores más importante son los pájaros y en menor grado los insectos (Ramírez \& Kallarackal, 2017). Los pájaros no solamente polinizan sino también comen toda la corola lo que evita la proliferación de Botrytis cinerea (Quintero, 2012). En Colombia, el agente ornitólogo más efectivo es la mirla negra (Turdus fuscater) (Fischer \& Parra-Coronado, 2020), mientras que en Nueva Zelanda son los Turdus merula y Acridotheres tristis, y en Brasil son los Turdus spp., Thraupis spp. y Tangara spp. (Duarte \& Paull, 2015). Estudios de polinización manual con pincel en Nueva Zelanda aumentaron el porcentaje de frutos cuajados, su calidad y peso en la variedad Apollo y disminuyeron el tiempo hasta su madurez en comparación con la polinización realizada por pájaros (Patterson, 1989). 


\section{Desarrollo, madurez y cosecha de los frutos}

En el caso de la propagación por medios vegetativos, la planta de feijoa inicia su producción en el tercer año, mientras que empleando el amarre de las ramas a posición horizontal, lo que debe iniciarse a partir de los 18 meses, los primeros frutos se pueden recolectar a partir del segundo año (Fischer, 2003). Morley-Bunker (2003) afirma que un huerto de feijoa de 2 a 4 años puede dar un rendimiento entre 4 y $5 t^{\text {ha- }}{ }^{-1}$, pero un huerto adulto tiene rendimientos de 20 a $25 \mathrm{t} \mathrm{ha}^{-1} \mathrm{o}$ entre 30 y $40 \mathrm{~kg}$ por árbol (Thorp, 2008). En el caso de una producción demasiado abundante, Duarte y Paull (2015) recomiendan un raleo de frutos pequeños o deformados, muy pronto después de la floración, lo que aumenta el tamaño y la calidad de los frutos restantes. En condiciones ambientales y de manejo favorables, los cultivares fértiles de feijoa pueden producir durante 30 años o más (Fischer, 2003).

Galvis (2003) observó que el desarrollo del fruto procedente de Tibasosa (Boyacá, Colombia), referente al peso fresco y seco o diámetro longitudinal y transversal, sigue una curva sigmoidal simple como ocurre en otros frutos como el lulo (Almanza-Merchán et al., 2016). El fruto de feijoa crece muy lento durante los primeros 40 días y mucho más acelerado a partir de los 50 días hasta su madurez fisiológica (Galvis, 2003).

El fruto, de acuerdo a la variedad, alcanza la madurez de recolección entre 120 y 150 días desde la floración (Quintero, 2012), sin embargo, es difícil identificar el grado de madurez apropiado porque se presentan cambios tenues en la composición química, las disminuciones en la firmeza y la tonalidad del color verde de la piel (Clark et al., 2005, Parra-Coronado et al., 2015b).

Es para tener en cuenta que una fruta cosechada antes de la madurez fisiológica -en la cual alcanza su máximo crecimiento y desarrollo- ya no desarrolla plenamente el sabor y la textura de la variedad (Parra \& Fischer, 2013); del mismo modo, permitir que el fruto caiga naturalmente al suelo significaría una pérdida de calidad y se clasificaría apenas como calidad industrial (Quintero, 2014).

Schotsmans et al. (2011) describen índices de madurez en la feijoa como (1) prueba de consistencia, la cual se prueba en la zona ecuatorial del fruto y si está suave se clasifica como apto para la recolección, (2) prueba de abscisión a través de la resistencia del pedúnculo y si se separan las células en la capa de abscisión, el fruto se desprende y es apto para la cosecha. En este fruto verde, las variaciones en la tonalidad de color de la piel, así como su forma y tamaño (dependiendo de la variedad) sirven también para indicar el momento de cosecha oportuno (ParraCoronado \& Fischer, 2013), sin embargo, exigen una gran experiencia del cosechador, por lo cual Quintero (2012) manifiesta que la cosecha de este fruto es mucho más compleja que la de otros frutales.

Después de la cosecha, las frutas maduran desde el centro hacia afuera; en el caso de frutos sobremaduros, ellos pierden el sabor por la reducción de los sólidos solubles y la acidez, así como por el oscurecimiento de la pulpa y las semillas (Parra-Coronado \& Fischer, 2013).

\section{Conclusiones}

De lo expuesto en esta revisión es evidente que la feijoa es un frutal con gran potencial para el consumo humano.

En Colombia se desarrolla en zonas entre el clima frío y frío moderado (1800 a $2700 \mathrm{msnm}$ ), resistiendo por corto tiempo temperaturas por debajo de $0^{\circ} \mathrm{C}$, pero las muy calientes afectan su floración; además, necesita solamente 
$3.04{ }^{\circ} \mathrm{C}$ como temperatura base para las fases iniciales de su etapa reproductiva.

La feijoa no es exigente con la calidad del suelo, pero enmiendas con materia orgánica y cal dolomita favorecen el desarrollo del árbol. Por el crecimiento relativamente lento del árbol, la aplicación de fertilizantes de lenta liberación de nutrientes también es beneficiosa.

En la poda prevalece la formación de copas con tres niveles de ramas agobiadas y la eliminación de las rectas, lo que fomenta la floración y la longevidad del árbol.

Existen muy pocos estudios fisiológicos en la feijoa que miden una tasa fotosintética relativamente baja comparada con otros frutales, pero con altos gastos energéticos durante la floración. La transpiración y el número de estomas siempre fueron mayores en el estrato alto de la copa que en el inferior y fueron fomentados por el aumento de la temperatura y la irradiancia, y la disminución de la humedad relativa en el árbol.

Las condiciones secas, así como una fertilización rica en fósforo, fomentan la inducción de flores, la floración y el cuajamiento de frutos. La polinización cruzada a través de pájaros como la mirla negra en Colombia fomenta la producción.

El fruto demora entre 120 y 150 días para su desarrollo y presenta una curva de crecimiento sigmoidal que dificulta la determinación del índice de madurez por su color verde, pero la producción por hectárea puede pasar las 20 t y entre 30 y 40 kg/árbol.

\section{Referencias}

Agronet. (2020). Producción nacional por producto. https://www.agronet.gov.co/estadistica/Paginas/home.. spx?cod=1.

Almanza-Merchán, P., Velandia, D. J. \&. Tovar, Y. (2016). Propiedades fisicoquímicas durante el crecimiento y desarrollo de frutos de lulo (Solanum quitoense Lam.). Rev. Colomb. Cienc. Hortic., 10(2), 222-231. https://doi.org/10.17584/rcch.2016v10i2.5065

Amarante, C. V. T. \& Santos, K. L. (2011). Feijoa (Acca sellowiana). Revista Brasileira de Fruticultura, 33(1).

Amarante, C. V. T., Souza, A. G., Beninca, T. D. T., Steffens, C. A. \& Ciotta, M. N. (2019). Physicochemical Attributes and Functional Properties of Flowers of Brazilian Feijoa Genotypes. Pesquisa Agropecuaria Brasileira, 54, e00445. https://doi.org/10.1590/S1678-3921.pab2019.v54.00445

Arias-Cruz, M. H., Velásquez, D., Mateus, H. Chaparro, Z. \& J. Orduz-Rodríguez. (2016). El rambután (Nephelium lappaceum), frutal asiático con potencial para Colombia: avances de la investigación en el piedemonte del Meta. Revista Colombiana de Ciencias Hortícolas, 10(2), 262-272. http://dx.doi.org/10.17584/rcch.2016v10i2.5761

Barrero, F. (1993). La ecotecnología en el contexto de la extensión y el desarrollo rural. Agro-Desarrollo, 4(1-2), 104117. 
Buitrago, S. D. (2017). Estudio de síntomas y crecimiento de plantas de feijoa (Acca sellowiana [O. Berg] Burret) en respuesta a los elementos faltantes $N, P, K, C a, M g$ y $B$. (Trabajo de grado). Facultad de Ciencias Agrarias, Universidad Nacional de Colombia. Bogotá, Colombia.

California Rare Fruit Growers Inc. -CRFG-. (1996). Feijoa. http://www.crfg.org/pubs/ff/feijoa.html.

Casierra-Posada, F. \& Fischer, G. (2012). Poda de árboles frutales. En G. Fischer (ed.), Manual para el cultivo de frutales en el trópico (pp. 169-185). Produmedios.

Casierra-Posada, F. \& Rodríguez, F. A. (2006). Tolerancia de plantas de feijoa (Acca sellowiana [Berg] Burret) a la salinidad por NaCl. Agronomía Colombiana, 24(2), 258-265.

Clark, C. J., White, A., Woolf, A. \& Domijan, K. 2005. Can Density Sorting at Harvest Segregate Mixed Maturity of Feijoa? Acta Horticulturae, 687, 93-98. http://dx.doi.org/10.17660/ActaHortic.2005.687.10Combariza, L.F., Neira, C. A.,

Fischer, G., Corredor, G. \& Quintero, O. C. (2007). Crecimiento, producción y calidad de fruta en feijoa [Acca sellowiana (O.Berg) Burret] en respuesta al nitrato de potasio, fosfato de potasio y ethephon. Revista Colombiana de Ciencias Hortícolas, 1(2), 170-181.

Donazzolo, J., Turra, E. L. C., Voss, L. C., Danner, M. A. Citadin, I. \& Nodari, R. O. (2019). Reproductive Biology and Flowering of Feijoa (Acca sellowiana (Berg) Burret) in Areas of Marginal Occurrence. Journal of Agricultural Science, 11(8). https://doi.org/10.5539/JAS.V11N8P156

Duarte, O. \& Paull, R. E. (2015). Exotic Fruits and Nuts of the New World. CABI Publishing.

Farias, D. P., Neri-Numa, I. A., Araújo, F. F. \& Pastore, G. M. (2020). A Critical Review of Some Fruit Trees from the Myrtaceae Family as Promising Sources for Food Applications with Functional Claims. Food Chemistry, 306, 125630. https://doi.org/10.1016/j.foodchem.2019.125630

Fischer, G. (2003). Ecofisiología, crecimiento y desarrollo de la feijoa. En G. Fischer, D. Miranda, G. Cayón \& M. Mazorra (eds.). (2003). Cultivo, poscosecha y exportación de la Feijoa (Acca sellowiana Berg) (pp. 9-26). Bogotá, Colombia: Produmedios.

Fischer, G. \& Melgarejo, L. M. (2020). The Ecophysiology of Cape Gooseberry (Physalis peruviana L.) - An Andean Fruit Crop. A Review. Revista Colombiana de Ciencias Hortícolas, 14(1). https://doi.org/10.17584/rcch.2020v14i1.10893

Fischer, G., Melgarejo, L. M. \& Cutler, J. (2018). Pre-harvest Factors that Influence the Quality of Passion Fruit: A Review. Agronomía Colombiana, 36(3), 217-226. http://dx.doi.org/10.15446/agron.colomb.v36n3.71751

Fischer, G. \& Orduz-Rodríguez, J. O. (2012). Ecofisiología en frutales. En G. Fischer (ed.), Manual para el cultivo de frutales en el trópico (pp. 54-72). Produmedios.

Fischer, G., Ramírez, F. \& Almanza-Merchán, P. J. (2012). Inducción floral, floración y desarrollo del fruto. En G. Fischer (ed.), Manual para el cultivo de frutales en el trópico (pp. 120-140). Produmedios.

Fischer, G. \& Parra-Coronado, A. (2020). Influence of Environmental Factors on The Feijoa (Acca sellowiana [Berg] Burret) Crop. A Review. Agronomía Colombiana, 38 (en imprenta). 
Gallo, Y., Toro, L.F., Jaramillo, H., Gutiérrez, P.A. \& Marín, M. (2018). Identificación y caracterización molecular del genoma completo de tres virus en cultivos de lulo (Solanum quitoense) de Antioquia (Colombia). Revista Colombiana de Ciencias Hortícolas, 12(2), 281-292. http://dx.doi.org/10.17584/ rcch.2018v12i2.7692.

Galvis, A. (2003). Manejo de la cosecha y poscosecha de la feijoa. En G. Fischer, D. Miranda, G. Cayón \& M. Mazorra (eds.). (2003), Cultivo, poscosecha y exportación de la Feijoa (Acca sellowiana Berg) (pp. 111-123). Produmedios.

García, O. J., Dueñez, E. Y., Fischer, G., Chaves, B. \& Quintero, O. C. (2008a). Efecto del nitrato de potasio, fosfato de potasio y ethephon en la inducción floral de la feijoa o goiabeira serrana (Acca sellowiana [0. Berg] Burret). Revista Brasileira de Fruticultura, 30(3), 577-584.

Garcia, O. J., Dueñez, E. Y., Fischer, G., Chaves, B. \& Quintero, O. C. (2008b). El cuajamiento frutos de feijoa (Acca sellowiana [O. Berg] Burret) en respuesta al nitrato de potasio, fosfato de potasio y ethephon. Agronomía Colombiana, 26(2), 217-225.

Germanà, C. \& Continella, A. (2004). Physiological Behavior of Some Subtropical Species in Mediterranean Area. Acta Horticulturae, 632, 117-123. https://doi.org/10.17660/ActaHortic.2004.632.15

Jackson, D. I. \& Looney, N. E. (1999). Temperate and Subtropical Fruit Production. CABI Publishing.

Martínez-Vega, R. R., Fischer, G., Herrera, A., Chaves, B. \& Quintero, O. C. (2008). Características físico-químicas de frutos de feijoa influenciadas por la posición en el canopi. Revista Colombiana de Ciencias Hortícolas, 2(1), 21-32.

Mitra, S. K. (2010). Important Myrtaceae Fruit Crops. Acta Horticulturae, 849, 33-38. https://doi.org/10.17660/ActaHortic.2010.849.2

Morley-Bunker, M. (1999). Feijoas. In D. I. Jackson \& N. E. Looney (eds.), Temperate and Subtropical Fruit Production (pp. 267-269) (2nd ed.). CABI Publishing.

Naizaque, J., García, G., Fischer, G. \& Melgarejo, L. M. (2014). Relación entre la densidad estomática, transpiración y las condiciones ambientales en feijoa (Acca sellowiana [O. Berg] Burret). Revista UDCA Actualidad y Divulgación Científica, 17(1), 115-121. https://doi.org/10.31910/rudca.v17.n1.2014.946

Pachón, G. \& Quintero, O. (1992). La feijoa (Feijoa sellowiana Berg) fruta promisoria para Colombia. Acta Horticulturae, 310, 239-248.

Parra-Coronado, A. \& Fischer, G. (2013). Maduración y comportamiento poscosecha de la feijoa (Acca sellowiana (O. Berg) Burret). Una revisión. Revista Colombiana de Ciencias Hortícolas, 7(1), 98-110. https://doi.org/10.17584/rcch.2013v7i1.2039

Parra-Coronado, A., Fischer, G. \& Chaves-Cordoba, B. (2015a). Tiempo térmico para estados fenológicos reproductivos de la feijoa (Acca sellowiana (O. Berg) Burret). Acta Biológica Colombiana, 20(1), 167-177. http://dx.doi.org/10.15446/abc.v20n1.42756

Parra-Coronado, A., Fischer, G. \& Camacho-Tamayo, J. H. (2015b). Development and Quality of Pineapple Guava Fruit in Two Locations with Different Altitudes in Cundinamarca, Colombia. Bragantia, 74(3), 359-366. https://doi.org/10.1590/1678-4499.0459 
Parra-Coronado, A., Fischer, G. \& Camacho-Tamayo, J. H. (2018). Post-harvest Quality of Pineapple Guava [Acca sellowiana (O. Berg) Burret] Fruits Produced in Two Locations at Different Altitudes in Cundinamarca, Colombia.

Agronomía Colombiana 36(1), 68-78. http://dx.doi.org/10.15446/agron.colomb.v36n1.68577

Parra-Coronado, A., Fischer, G. \& Camacho-Tamayo, J. H. (2019). Influencia de las condiciones climáticas de cultivo en la calidad en cosecha y en el comportamiento poscosecha de frutos de Feijoa. Tecnología en Marcha, 32, (Especial XIII CLIA 2018), 86-92.

Patterson, K. J. (1989). Effect of Hand Pollination on Fruit Set and Fruit Quality of the Feijoa (Acca sellowiana). Acta Horticulturae, 240, 197-200.

Peña, F. J. \& Cabezas, M. (2014). Aspectos ecofisiológicos de la feijoa (Acca sellowiana Berg) bajo condiciones de riego y déficit hídrico. Revista UDCA Actualidad \& Divulgación Científica, 17(2), 381-390.

Perea, M., Fischer, G. \& Miranda, D. (2010). Feijoa Acca sellowiana Berg. En M. Perea, L.P. Matallana \& A. Tirado (eds.), Biotecnología aplicada al mejoramiento de los cultivos de frutas tropicales (pp. 330-349). Universidad Nacional de Colombia, Facultad de Ciencias.

Quintero, O. (1993). Control de las cosechas empleando nuevas técnicas de poda en germoplasma y cultivares seleccionados de feijoa (Acca sellowiana). Agro-Desarrollo, 4(1-2), 89-106.

Quintero, O. (2003). Selección de cultivares, manejo del cultivos y regulación de cosechas de feijoa. En G. Fischer, D. Miranda, G. Cayón \& M. Mazorra (eds.). (2003), Cultivo, poscosecha y exportación de la Feijoa (Acca sellowiana Berg) (pp. 49-71). Produmedios.

Quintero, O. C. (2012). Feijoa (Acca sellowiana Berg). En G. Fischer (ed.), Manual para el cultivo de frutales en el trópico (pp. 443-473). Produmedios,

Quintero, O. C. (2014). La feijoa en Colombia. En Memorias VI Encontro sobre Pequenas Frutas e Frutas Nativas do Mercosul (pp. 167-178). Empraba Clima Temperado.

Ramírez, F. \& Kallarackal, J. (2017). Feijoa [Acca sellowiana (O. Berg) Burret] Pollination: A Review. Scientia Horticulturae, 226, 333-341. https://doi.org/10.1016/j.scienta.2017.08.054

Ramírez, F. \& Kallarackal, J. (2018). Phenological Growth Stages of Feijoa [Acca sellowiana (O. Berg) Burret] According to the BBCH Scale under Tropical Andean Conditions. Scientia Horticulturae, 232, 184-190. https://doi.org/10.1016/j.scienta.2017.12.059

Sachet, M. R., Citadin, I., Guerrezi, M. T., Pertille, R. H., Donazzolo, J. \& Nodari, R. O. (2019). Non-destructive Measurement of Leaf Area and Leaf Pigments in Feijoa Trees. Revista Brasileira de Engenharia Agrícola e Ambiental, 23(1), 16-20. http://dx.doi.org/10.1590/1807-1929/agriambi.v23n1p16-20

Sánchez-Mora, F. D., Saifert, L., Pasa, M. S., Ciotta, M. N., Ribeiro, H. N., Rojas-Molina, A., Lombardi, G. \& Nodari, R. O. (2017). Poda de frutificação em variedades comerciais de goiabeira-serrana. p. 73. En XV Encuentro Nacional de Fruticultura de Clima Temperado. 25-27 de julio de 2017. Fraiburgo (SC), Brasil.

Sánchez-Reinoso, A. D., Jiménez-Pulido, Y., Martínez-Pérez, J. P., Pinilla, C. S, \& Fischer, G. (2019). Chlorophyll 
Fluorescence and other Physiological Parameters as Indicators of Waterlogging and Shadow Stress in Lulo (Solanum quitoense var. septentrionale) Seedlings. Revista Colombiana de Ciencias Hortícolas, 13(3), 325-335. https://doi.org/10.17584/rcch. 2019v13i3.100171

Schotsmans, W. C., East, A., Thorp, G. \& Woolf, A. B. (2011). Feijoa (Acca sellowiana [Berg] Burret). In E.M. Yahia (ed.) Postharvest Biology and Technology of Tropical and Subtropical Fruits (pp. 115-133). Vol. 3. Woodhead Publishing.

Sharpe, R. H., Sherman, W. B. \& Miller, E. P. (1993). Feijoa, History and Improvement. Proceedings of the Florida State Horticultural Society, 106, 134-139.

Thorp, G. (2008). Feijoa Acca sellowiana (Berg) Burret, Myrtaceae. In J. Janick \& R. E. Paulll (eds.), Encyclopedia of Fruit and Nuts (pp. 526-533). CAB International.

Zhu, F. (2018). Chemical and Biological Properties of Feijoa (Acca sellowiana). Trends in Food Science \& Technology, 81, 121-131. https://doi.org/10.1016/j.tifs.2018.09.008

Cómo citar este artículo: Fischer, G., Parra-Coronado, A. \& Balaguera-López, H. (2020). Aspectos del cultivo y la fisiología de la feijoa (Acca sellowiana [Berg] Burret). Una revisión. Cien. Agri. 17(3), 11-24. https://doi.org/10.19053/01228420.v17.n3.2020.11386 